\title{
Cardiac tamponade as a presentation of COVID-19 after cardiac surgery
}

\author{
Kirun Gopal ${ }^{1}\left[\right.$ ] Hisham Ahamed ${ }^{2} \cdot$ Rajesh Jose $^{1} \cdot$ Praveen Kerala Varma $^{1}$ (]
}

Received: 15 July 2021 / Revised: 8 September 2021 / Accepted: 9 September 2021 / Published online: 23 November 2021

(c) Indian Association of Cardiovascular-Thoracic Surgeons 2021

\begin{abstract}
We present two patients who turned positive for coronavirus disease 2019 (COVID-19) after elective cardiac surgery, developing postoperative pericardial effusion with tamponade. One of the patients who presented with tamponade had no other symptoms of COVID-19 infection. COVID-19 can rarely present with cardiac manifestations including pericardial effusion and tamponade. In the post cardiac surgery setting, this effect of the virus may be accentuated. Hence, a high index of suspicion and prompt management are the keys for a successful outcome.
\end{abstract}

Keywords COVID-19 $\cdot$ Pericardial effusion $\cdot$ Tamponade $\cdot$ Cardiac surgery

\section{Introduction}

Postoperative pericardial effusion leading to tamponade is a known complication of cardiac surgery occurring in $0.1-6 \%$ of patients [1]. The current global pandemic due to coronavirus disease 2019 (COVID-19) rarely affects the heart compared to the lungs. Clinical presentation primarily with cardiac involvement without preceding fever and respiratory symptoms is rare. COVID-19 disease presenting as a de novo cardiac tamponade has only recently been described [2-4]. We present two patients who presented with cardiac tamponade after cardiac surgery, both of whom were diagnosed to have COVID-19 after surgery, and one of whom had no other symptoms suggestive of COVID-19.

Kirun Gopal

kirungopal@gmail.com

1 Department of Cardiovascular and Thoracic Surgery, Amrita Institute of Medical Sciences, Amrita Vishwa Vidyapeetham (Amrita University), Kochi, Kerala, India

2 Department of Cardiology, Amrita Institute of Medical Sciences, Amrita Vishwa Vidyapeetham (Amrita University), Kochi, Kerala, India

\section{Case reports}

\section{Patient 1}

A 40-year-old male with coronary artery disease with normal left ventricular ejection fraction (LVEF 60\%) underwent off pump coronary artery bypass graft surgery (OPCAB). His immediate postoperative recovery was uneventful, and he was shifted out of the intensive care unit (ICU) on day 2. Routine transthoracic echocardiogram on day 5 showed moderate size pericardial effusion with early signs of tamponade and global biventricular dysfunction (Figs. 1 and 2). A twelve-lead electrocardiogram showed characteristic findings of pericarditis such as concave upwards modest ST elevation in chest and limb leads and reciprocal ST depression and PR elevation in aVR (Fig. 3). As the patient was asymptomatic, it was decided to keep the patient nil-by-mouth for 3 to $4 \mathrm{~h}$ before induction of general anesthesia. He however developed a gradual onset of breathlessness and was shifted to the ICU. He was electively intubated. Subsequently, while inserting central and arterial lines in preparation for surgery, he had a cardiac arrest. Cardiopulmonary resuscitation was initiated without immediate return of spontaneous circulation. The chest was opened immediately in the ICU, and blood-stained pericardial fluid was drained. Internal massage was given, and the cardiac rhythm was restored with acceptable hemodynamics with inotropic support. During the time of the internal cardiac massage, it was noticed that the heart felt very warm and the heart surface appeared red 

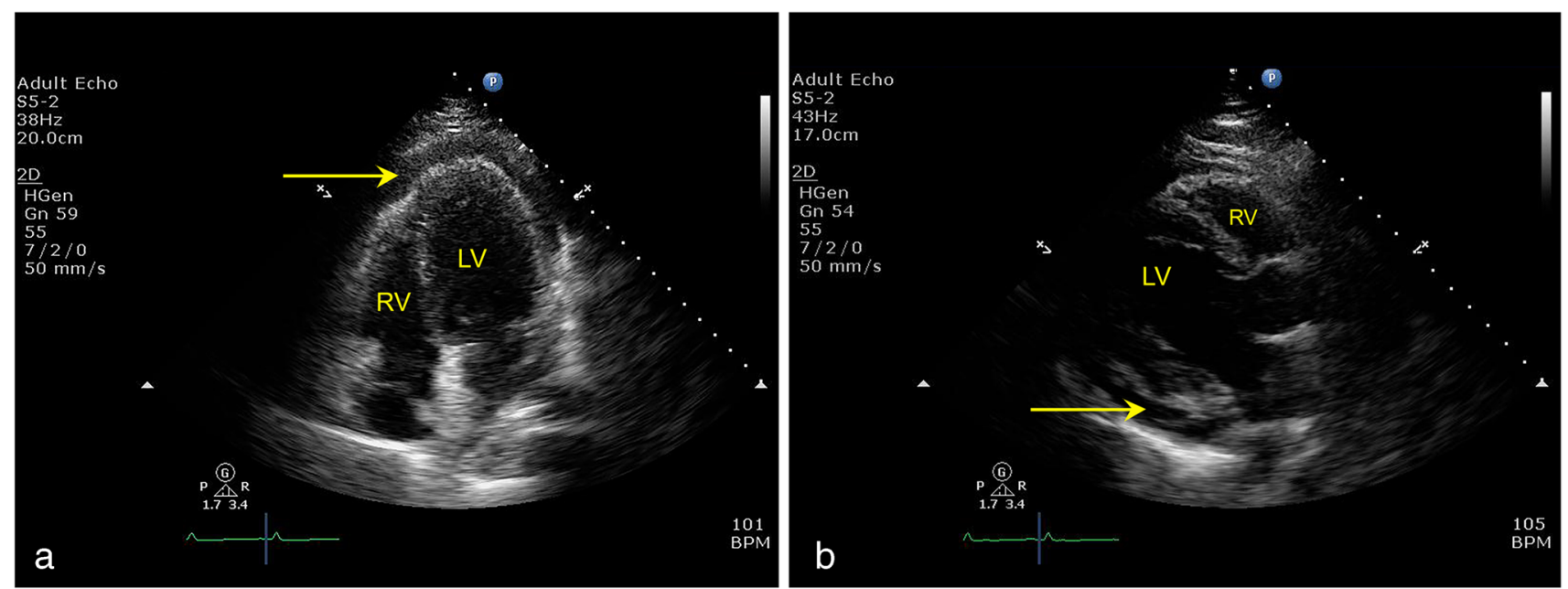

Fig. 1 a Echocardiogram four chamber view showing circumferential pericardial effusion—arrow. LV, left ventricle. RV, right ventricle. b Parasternal long axis view showing effusion—arrow. LV, left ventricle. RV, right ventricle
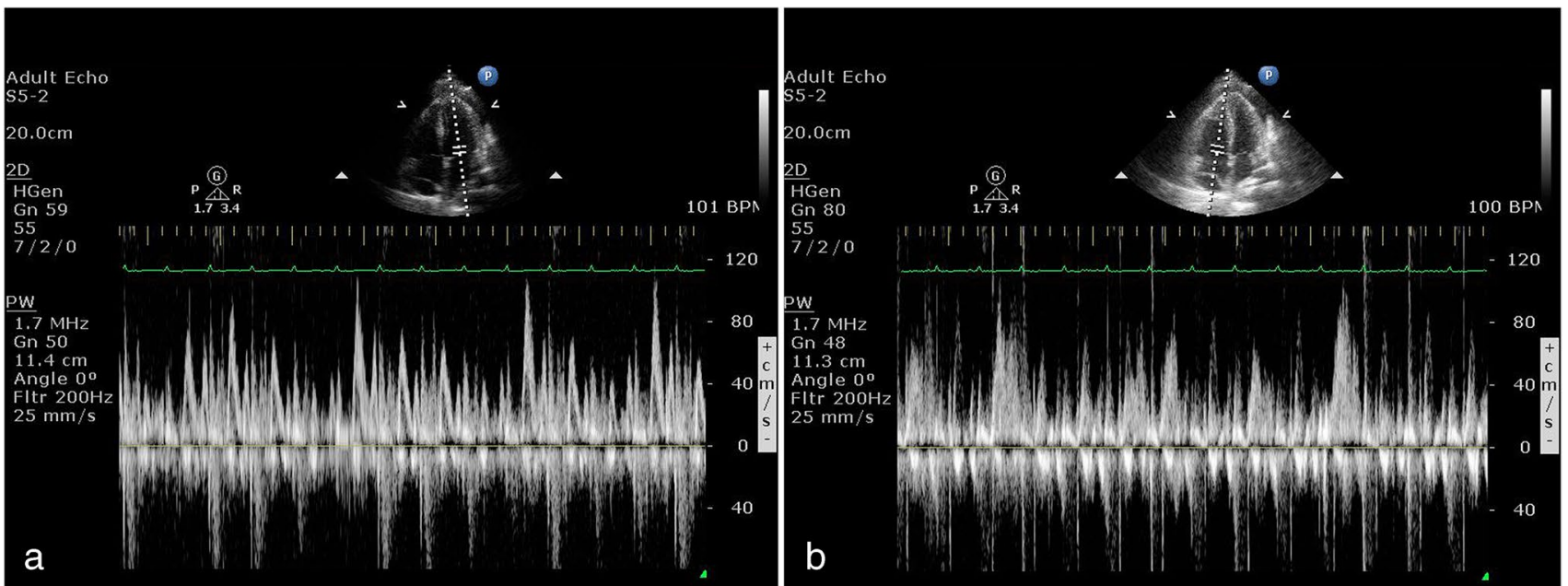

Fig. 2 a, b Mitral and tricuspid inflow Doppler showing features consistent with early tamponade in the setting of ventricular dysfunction

and inflamed. All the grafts appeared patent on inspection and were pulsatile on palpation, nor were there any new areas of regional wall motion abnormality on echocardiogram to suggest graft occlusion. He maintained stable hemodynamics with inotropic support. However, he was noticed to be febrile at this time. In view of the fever, and the appearance of the warm and inflamed heart, a repeat reverse transcriptase polymerase chain reaction (RT-PCR) test for severe acute respiratory syndrome coronavirus 2 (SARSCoV-2) was sent from the ICU, which came back positive. He had tested negative for COVID-19 preoperatively. As part of the stratification of his COVID-19 disease, inflammatory markers were sent which were very high, with serum ferritin at $195,321 \mathrm{ng} / \mathrm{ml}$ (ref range 30-400), serum lactate dehydrogenase 25,540 U/L (ref range 0-248), and D-Dimer
$8.03 \mu \mathrm{g} / \mathrm{ml}$ (ref rang $0-0.5$ ). He was started on intravenous remdesivir and dexamethasone for the COVID-19 infection. He however subsequently developed multi-organ dysfunction, with renal and hepatic failure, and deteriorated over the next 2 days and expired $36 \mathrm{~h}$ after the event.

\section{Patient 2}

A 49-year-old patient underwent elective OPCAB. On postoperative day 3 , he developed fever and a drop in saturation. A repeat RT-PCR for SARS-CoV-2 was sent which came back positive. He had tested negative preoperatively. He subsequently was shifted to the Covid ICU. His inflammatory markers were high with serum ferritin at $2166 \mathrm{ng} / \mathrm{ml}$, lactate dehydrogenase $292 \mathrm{U} / \mathrm{L}$, and D-Dimer $3.95 \mu \mathrm{g} / \mathrm{ml}$. He was started 


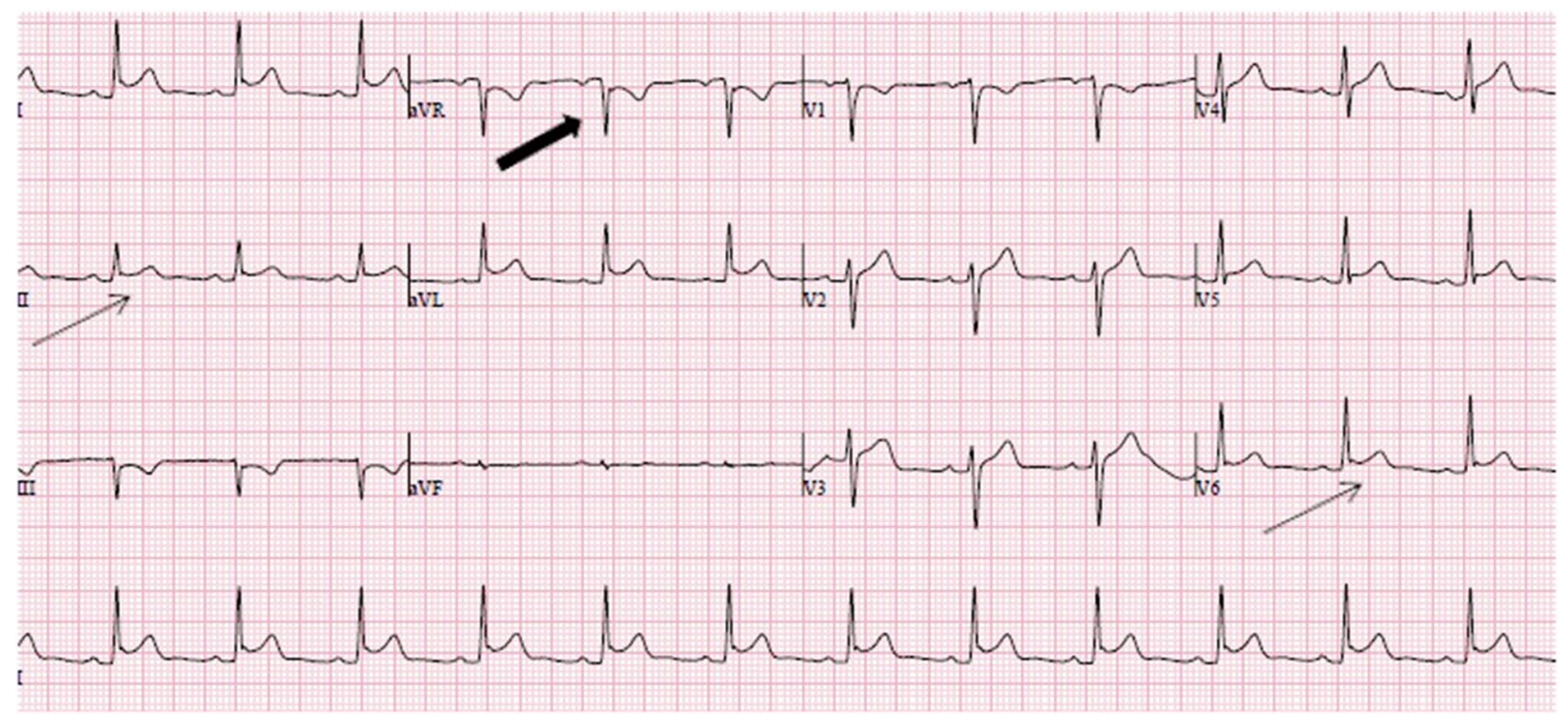

Fig. 3 Electrocardiogram showing characteristic changes of pericarditis with diffuse concave upwards ST segment elevation in most of the leads (line arrow) and ST segment depression in aVR (block arrow)

on intravenous remdesivir and dexamethasone by the infectious disease team. He was stable for 2 days with supplemental oxygen but then developed worsening tachypnea and hypotension. He was started on inotropic support and electively intubated with the provisional diagnosis of systemic inflammatory response syndrome, related to the cytokine storm associated with COVID-19 disease. At that time, a bedside echocardiogram was done which showed a significant pericardial effusion with features of tamponade. He was immediately shifted to the operating room, and the pericardial effusion was drained. The bypass grafts appeared patent and were pulsatile on palpation. The surface of his heart appeared red and inflamed at the time of re-exploration. He subsequently made a slow but steady recovery, and pre discharge echocardiogram showed no residual or recurrent pericardial effusion. He was discharged on dual antiplatelets and other routine cardiac medication and multivitamins. As there was no residual effusion, we did not put him on anti-inflammatory agents or non-steroidal antiinflammatory medication at discharge. The patient is doing well on follow-up 8 months after the procedure, with no obvious long-term effects of COVID-19, and with subsequent echocardiograms showing no recurrent pericardial effusion and normal ventricular size and function.

\section{Discussion}

COVID-19 is a viral disease caused by the novel coronavirus, SARS-COV-2. The disease primarily affects the respiratory system presenting with fever, cough, and shortness of breath. More rarely COVID-19 can present with cardiovascular symptoms. Cardiovascular manifestations of COVID-19 include myocarditis, arrhythmias, acute coronary syndrome, venous thromboembolism, cardiomyopathy, and heart failure [5]. It is now known that the presence of cardiovascular disease can predispose a person to COVID19 and also to be at elevated risk of adverse outcomes from the infection. The converse is also true with COVID-19 increasing the risk for cardiovascular complications [6, 7]. Viruses as a cause of myocarditis are well known, especially by influenza and parvovirus B-19 [8]. Myocarditis causes localized or generalized myocardial inflammation and necrosis leading to ventricular dysfunction [8]. Myocarditis can present as ventricular dysfunction as seen in our first patient who had normal ventricular function preoperatively [4]. Pericardial involvement due to COVID-19 has been more rarely reported and is probably related to the systemic inflammatory response associated with the disease related to direct cytotoxic activity of the virus or immunemediated pathways $[2,3]$. The incidence of pericardial effusion associated with COVID-19 infection is around 5\% and maybe a sign of more severe inflammatory response [9]. There have been reports in the literature of isolated presentation of COVID-19 as cardiac tamponade, which were treated with pericardiocentesis or pericardial window $[2,3]$. One characteristic noted in these patients was the presence of a hemorrhagic pericardial effusion. Other causes of hemorrhagic pericardial effusions include malignant effusion, infection-related effusion, post trauma or surgery effusion, and effusion associated with bleeding disorders [10]. The 
two patients in this report, being post cardiac surgery, had hemorrhagic effusions, and any independent contribution of the COVID-19 disease was difficult to ascertain. We believe that the warm, red, and inflammatory appearance different from the usual appearance of post cardiac surgery tamponade hearts may be suggestive of the post COVID-19 inflammatory state. Prior reports attempting to attribute causation of pericardial effusion to COVID-19 disease by isolating the virus in the fluid by RT-PCR has not been widely proven due to non-standardization of the technique and the variability in reporting $[2,3]$. The pathogenic mechanism of hemorrhagic effusion in relation to COVID-19 is yet to be elucidated. However, there are no reports of post cardiac surgery presentation of tamponade associated with COVID-19. One of the mechanisms involved is in all likelihood similar to patients with COVID-19 who presented with de novo cardiac tamponade without any history of surgery, attributed to the inflammatory effects of the virus leading to pericarditis and subsequent effusion, and in all probability exacerbated by the post cardiac surgery status. Though it is not possible to show direct causation between COVID-19 and cardiac tamponade in these 2 patients, in light of the propensity for COVID-19 to cause pericardial effusion, it is prudent for cardiac surgeons to be aware of this possible association.

\section{Conclusion}

In these times of COVID-19 and its associated negative impact on the cardiovascular system, it is imperative for cardiac surgeons to be aware of the rare presentation of cardiac tamponade in COVID-19, especially in susceptible patients such as those after cardiac surgery.

\section{Funding None.}

\section{Declarations}

Ethics committee approval Not required.

Consent for publication As individual patient data was anonymized, individual patient consent for publication was not obtained.

Conflict of interest The authors declare no competing interests.
Human and animal rights statement All procedures performed in this study involving human participants were in accordance with the ethical standards of the institutional research committee and with the 1964 Helsinki Declaration and its later amendments or comparable ethical standards. This article does not contain any studies with animals performed by any of the authors.

\section{References}

1. Leiva EH, Carreño M, Bucheli FR, Bonfanti AC, Umaña JP, Dennis RJ. Factors associated with delayed cardiac tamponade after cardiac surgery. Ann Card Anaesth. 2018;21:158-66. https://doi. org/10.4103/aca.ACA_147_17.

2. Singh R, Fuentes S, Ellison H, et al. A case of hemorrhagic cardiac tamponade in a patient with COVID-19 infection. CASE (Phila). 2020;4:316-9.

3. Dabbagh MF, Aurora L, D'Souza P, Weinmann AJ, Bhargava P, Basir MB. Cardiac tamponade secondary to COVID-19. JACC Case Rep. 2020;2:1326-30.

4. Kogler W, Omar M, Maaliki N, Patel S, Foldy S, Ruiz J. A case series of precipitous cardiac tamponade from suspected perimyocarditis in COVID-19 patients. J Geriatr Cardiol. 2021;18:403-6. https://doi.org/10.11909/j.issn.1671-5411.2021.05.001.

5. Driggin E, Madhavan MV, Bikdeli B, et al. Cardiovascular considerations for patients, health care workers, and health systems during the COVID-19 pandemic. J Am Coll Cardiol. 2020;75:235271. https://doi.org/10.1016/j.jacc.2020.03.031.

6. Li B, Yang J, Zhao F, et al. Prevalence and impact of cardiovascular metabolic diseases on COVID-19 in China. Clin Res Cardiol. 2020;109:531-8. https://doi.org/10.1007/s00392-020-01626-9.

7. Zheng Y-Y, Ma Y-T, Zhang J-Y, Xie X. COVID-19 and the cardiovascular system. Nat Rev Cardiol. 2020;17:259-260. https://doi. org/10.1038/s41569-020-0360-5.

8. Inciardi RM, Lupi L, Zaccone G, et al. Cardiac involvement in a patient with coronavirus disease 2019 (COVID-19). JAMA Cardiol. 2020;5:819-24. https://doi.org/10.1001/jamacardio.2020. 1096.

9. Ye Z, Zhang Y, Wang Y, Huang Z, Song B. Chest CT manifestations of new coronavirus disease 2019 (COVID-19): a pictorial review. Eur Radiol. 2020;30:4381-9.

10. Atar S, Chiu J, Forrester JS, Siegel RJ. Bloody pericardial effusion in patients with cardiac tamponade: is the cause cancerous, tuberculous, or iatrogenic in the 1990s? Chest. 1999;116:1564-9. https://doi.org/10.1378/chest.116.6.1564.

Publisher's note Springer Nature remains neutral with regard to jurisdictional claims in published maps and institutional affiliations. 